\title{
School-based Internet-tailored fruit and vegetable education combined with brief counselling increases children's awareness of intake levels
}

\author{
Resiti T Mangunkusumo ${ }^{1}$, Johannes Brug ${ }^{1}$, Harry J de Koning ${ }^{1}$, Johan van der Lei ${ }^{2}$ \\ and Hein Raat ${ }^{1, *}$ \\ 'Department of Public Health, Erasmus MC-University Medical Center Rotterdam, PO Box 2040, 3000 CA \\ Rotterdam, The Netherlands: ${ }^{2}$ Department of Medical Informatics, Erasmus MC-University Medical Center \\ Rotterdam, Rotterdam, The Netherlands
}

Submitted 22 March 2006: Accepted 9 August 2006

\begin{abstract}
Objective: Children's fruit/vegetable intake is still below recommended levels. This study applied Internet-tailored advice for schoolchildren and Internet-supported brief dietary counselling (with child and parent) within preventive health care to promote fruit/vegetable intake.

Setting/subjects: The study involved 30 seventh-grade classes (16 in the intervention group and 14 in the control group) with a total of 675 children aged 9-12 years, of whom 495 were allowed to participate.

Design: A cluster-randomised baseline-post-test experimental design was applied. During school hours, all children completed Internet-administered questionnaires on fruit/vegetable intake and related determinants. Children in the intervention group received immediate online individually tailored nutrition feedback. For each child in the intervention group, a nurse received information concerning the assessment of fruit/vegetable intake via the Internet to support a 5 min counselling protocol to promote fruit/vegetable intake. Children completed a similar post-test questionnaire 3 months after the first assessment. Intention-to-treat analyses were conducted using multilevel regression analyses.

Results: A total of 486 children (98\% of 495) participated (263 in the intervention group, 223 in the control group); 240 child-parent couples in the intervention group attended the counselling. Awareness of inadequate fruit intake (odds ratio $(\mathrm{OR})=3.0$; 95\% confidence interval $(\mathrm{CI})=1.8-5.3)$ and knowledge of recommended vegetable intake levels $(\mathrm{OR}=2.7$; $95 \% \mathrm{CI}=1.8-4.1)$ were significantly more likely at post-test in the intervention group than in the control group. No significant effects were found on intake or other determinants.

Conclusions: A compact, integrated two-component intervention can induce positive changes in knowledge and awareness of intake levels of fruit/vegetables among schoolchildren. To induce changes in intake levels, more comprehensive interventions may be needed.
\end{abstract}

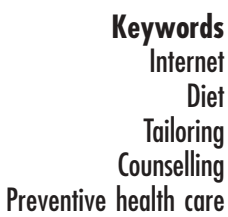

Sufficient intake of fruits and vegetables may contribute to the prevention of cardiovascular diseases, certain cancers and obesity ${ }^{1-4}$. Across Europe, including The Netherlands, current intake levels among schoolchildren are below recommendations ${ }^{5}$. Adopting a health-promoting diet at a young age may help to establish healthy eating in later life ${ }^{6,7}$.

Various evidence-based interventions are available to encourage healthy dietary habits for schoolchildren, and tailoring of intervention messages is considered a promising approach for promoting healthy eating among young people ${ }^{8-10}$. In general (mainly targeting adults), computer-tailored nutrition education is more likely to be read, processed more intensively and appreciated better than more general intervention materials $^{11-13}$. It also appears to have a positive impact on behavioural change ${ }^{12,14}$. Since nurses (i.e. school nurses) already invite children for consultation as current practice in (Dutch) preventive health care, complementing this with computer-tailored nutrition advice and dietary counselling by a nurse may be an efficient approach in promoting a healthy diet. To our knowledge, studies regarding computer-tailored nutritional interventions for schoolchildren as well as the involvement of nurses in such school-based interventions are unreported. 
The present study aimed to evaluate the effectiveness of a two-component intervention on fruit/vegetable intake by comparing a group receiving the intervention with a control group. It also explored effects on potential determinants of intake levels and self-reported intake as important initial steps towards behavioural change. In addition, we determined the level of exposure to the intervention.

\section{Methods}

\section{Two-component intervention}

This study applied a school-based intervention among children aged 9-12 years. The intervention consisted of a combination of Internet-tailored nutrition advice for the children followed by Internet-supported brief dietary counselling by the nurse in the presence of at least one parent. This two-component intervention was informed by behavioural change theory and recent evidence that healthy behavioural change is more likely when children are aware of their inadequate intake levels, have knowledge of the recommended intake levels, like to eat fruit/vegetables, and when the fruit/vegetables they like are available and accessible $9,15-18$.

\section{Internet-tailored advice}

The Internet-tailored advice specifically aimed to increase knowledge of recommended intake levels, increase awareness of personal intake levels, and stimulate children's liking of fruit/vegetables and their availability in the home.

During school hours the children in both the intervention and control group completed Internet-administered questionnaires on fruit/vegetable intake and related determinants. Children in the intervention group received immediate online feedback about nutrition, individually tailored to each child's answers ${ }^{12,19}$. First, the advice explained the recommended intake levels in order to increase knowledge and then compared the child's personal intake with the recommendations in order to increase awareness. The daily recommendations were two pieces of fruit and $150 \mathrm{~g}$ (three 'serving spoons') of vegetables ${ }^{5}$. Second, the feedback encouraged the child to eat sufficient amounts of fruit/vegetables, e.g. by trying different types of fruit/vegetables and asking their parents to buy/give them the fruit/vegetables that they like.

Children were encouraged to take home a printout of the advice. The teachers supervised the completion of the questionnaire, as well as the printing out of the advice. The assessment took place in either the classroom or in the school's computer area (depending on the school facilities).

\section{Dietary counselling}

The dietary counselling focused on increasing the child's knowledge about recommended intake levels, increasing awareness of personal intake levels, and motivating the child to eat sufficient fruit/vegetables and/or discussing any barriers in order to increase self-efficacy for behavioural change. An additional aim of the counselling was to involve the child's home in the process of behavioural change.

To support the counselling, the nurse had access to the data on the child's intake and related determinants that were assessed with the computer-tailored Internet tool; a website provided the nurse with a summary of the child's dietary status and related determinants, the Internettailored advice and the child's answer to each item.

About two weeks after the Internet-tailored advice, the child together with one parent attended the brief dietary counselling. The nurse applied a protocol in order to promote the child's fruit/vegetable intake. First, the nurse tried to reinforce knowledge on recommendations and increase awareness of the child's inadequate intake. The focus of the remaining counselling time depended on the child's (and parent's) motivation to eat sufficient fruit/vegetables. If the motivation to change was lacking, then the nurse explained the benefits of adequate intake and tried to dispel misconceptions. If motivation was present, the nurse discussed possible barriers to eating sufficient fruit/vegetables and how to overcome these barriers, in some cases resulting in defining personal goals to achieve behavioural change. This approach is based on the Minimal Intervention Strategy as applied, for example, in counselling to cease smoking ${ }^{20}$.

In any case, the nurse would again give a printout of the Internet-tailored advice to the child and a leaflet on the importance of vegetable consumption to the parents. This counselling (which took about $5 \mathrm{~min}$ ) occurred at the end of the periodic health examination, which is a routine contact moment of the municipal health services with parents/guardians and their child. Thus children in both the intervention and control groups received the periodic health examination, but only children in the intervention group received the additional dietary counselling. The counselling took place in a private room either at school or at the health service's premises.

\section{Internet tool}

The Internet tool of the two-component intervention was developed using PHP (4.0.1 and higher), MySQL (3.22 and higher) and JavaScript (1.3). Access to the questionnaire and advice was password-protected. Data were sent to the server in a scrambled format. Logging out after completing the questionnaire was permitted only after answering all questions. Each nurse received a personal log-in code from the researcher to access the Internet tool and thereby the data for each child.

\section{Study design and sample}

A cluster-randomised baseline-post-test experimental design was applied ${ }^{21}$. Two participating municipal health 
services, one in a rural area (Harderwijk) and one in an urban area (Vlaardingen) in The Netherlands, invited 27 elementary schools from various socio-economic and religious backgrounds to participate. These schools comprised 30 classes from the seventh grade with 675 schoolchildren (aged 9-12 years) and all had adequate computer and Internet facilities. From the Vlaardingen region 14 classes participated, involving two nurses, and from the Harderwijk region 16 classes participated, involving three nurses. In each class all children received counselling from the same nurse, as this is the standard practice for municipal health services. After recruitment, the classes were randomly assigned ('within' nurses) to the intervention group with tailored nutrition advice and counselling (16 classes) or the control group (14 classes). This clustering was done to minimise contamination.

The parents and children, blinded to which group they belonged to, received information about the project and parents were allowed several weeks to actively give written consent for participation. This resulted in 495 (73\%) children with permission to participate. The Medical Ethical Committee of the Erasmus MC-University Medical Center Rotterdam approved the study.

\section{Evaluation measures}

All children completed (during school hours) an Internetadministered baseline questionnaire and about three months later a similar post-test questionnaire on potential determinants and intake related to fruit/vegetables, as well as questions on gender, age, type of family, and countries of birth of the child and parents to determine ethnic background $^{22}$. The baseline questionnaire was also the questionnaire used for the assessment of the Internettailored advice. After receiving the Internet-tailored advice, the children completed a brief evaluation form to assess the level of exposure to the intervention. The nurses did the same after giving the counselling.

\section{Primary outcome measures}

The primary outcome measures were fruit/vegetable intake. Children completed the fruit/vegetable intake questions that were derived from, and thus not exactly the same as (e.g. different categories), the fruit/vegetable food-frequency questionnaire (FFQ) validated by Van Assema et $a .^{23}$. The relative validity of the FFQ was assessed by comparison with 7-day dietary records. The correlations of the validity estimates ranged between 0.29 and 0.59 for citrus fruit and other fruit intake, and between 0.22 and 0.35 for total vegetable intake (heated and raw/ salad, last week).

In the present questionnaire children were asked about the number of days on which they ate fruit (such as apples, mandarins, pears, or oranges) during the week before FFQ administration and the number of pieces of fruit on such days; multiplying the frequency and quantity and dividing this by seven assessed the 'fruit frequency last week' in servings per day. A pre-coded 24-hour recall assessed vegetable intake of the day before administration. The recall was divided into three parts: heated vegetables, salad, and vegetables in mixed dishes. Each part started with a general question such as 'Did you eat heated vegetables (such as broccoli, French beans, and carrots) yesterday?' (yes/no), and if confirmed, followed by 'How much of such vegetables did you eat yesterday?' Answers ranged from one serving spoon to three or more serving spoons. Standards were defined for the units in spoons: one serving spoon of heated vegetables equalled $50 \mathrm{~g}$, one serving spoon of salad equalled $20 \mathrm{~g}$, and one serving spoon of vegetables in mixed dishes equalled $15 \mathrm{~g}$. Summing the grams of these three types of vegetables assessed the '24-hour recall vegetable intake'. Finally, confirmation of two dichotomous items, 'Do you eat fruit everyday during schooldays?' and 'Do you eat fruit every Saturday and Sunday?', established the number of children reporting 'usual daily intake' for fruit. This was also done for vegetables.

\section{Secondary outcome measures}

As secondary outcome measures, potential behavioural determinants of dietary intake were assessed for fruit and vegetables separately. One question measured whether the child thought that he/she ate a sufficient amount of fruit (in yes/no format). In combination with the child's actual intake (see above) being adequate or not, this determined the child's 'awareness' of inadequate intake for fruit in the previous week; the same was done for vegetable intake during the previous day. To assess 'knowledge' of recommended intake levels, children were asked (on an 8-point scale) how much fruit they should eat to have a healthy eating pattern. Response options ranged from no fruit to five pieces a day or more. This was subsequently re-coded into a dichotomous variable (amount too low vs. correct/higher amount); the same was done for recommended vegetable intake levels, dichotomised from response options ranging from no vegetables to five serving spoons $(\sim 250 \mathrm{~g})$ a day or more. Asking the children if they thought eating fruit every day was achievable or not assessed 'self-efficacy'. This was dichotomised from a 5-point Likert scale (negative or neutral scores $=0$; positive scores $=1$ ); the same was done for vegetable intake. A single item assessing the child's 'liking' of fruit was dichotomised from a 3-point Likert scale to the child reporting liking many types (negative or neutral scores $=0$; positive scores $=1$ ); the same was done for liking vegetables. The 'availability' of fruit was assessed by an item on how often fruit was available at home. Response options were dichotomised from a 3-point Likert scale to the child reporting they always had fruit at home (negative or neutral scores $=0$; positive scores $=1$ ). Finally, the number of types of vegetables available determined the 'availability' of vegetables at dinner on a 4-point scale. Response options 
ranged from usually having no vegetables to more than two types of vegetables. This was subsequently recoded into a dichotomous variable (less than two types vs. two or more types).

\section{Measures to assess exposure to the intervention}

After the Internet-tailored advice, the children completed an additional item on how much they had read of the advice, with four possible answers ranging from having read nothing to having read both the fruit and vegetable advice completely. After the counselling session, the nurses completed a form to assess the child's attendance at the counselling. Additionally, the nurses noted which of four topics they concluded the counselling session with: i.e. increasing motivation to change, increasing selfefficacy, setting goals, or sustaining/taking action to eat sufficiently.

\section{Statistical analysis}

To test for equality of groups at baseline, multiple logistic regression of treatment condition (yes/no) was conducted using SPSS version 11.0.1. Baseline measures and sociodemographic characteristics were the independent variables. The same regression was done for dropouts (yes/no), with baseline measures, sociodemographic characteristics and treatment condition as independent variables. This allowed us to identify potential correlates of dropout and thereby potential biases in post-test data analyses.

In all effect analyses, we included children with a missing post-test value due to dropout by replacing the missing post-test value with the baseline value (i.e. the last observation carried forward method, 'intention-to-treat analyses, $^{24}$ ).

Due to the study design, children were grouped within nurses and classes with a high probability of interdependence between children of the same class and/or of the same nurse. To take this into account, multilevel analyses with random intercepts were conducted in MLwiN $2.02^{25}$ to assess the effects of the intervention on behaviour and determinants of behaviour, while controlling for region (i.e. municipal health service), gender, age and baseline value of the outcome measures (except for knowledge and self-efficacy, for which no baseline measure had been assessed). The regression coefficient $(\beta)$ or odds ratio (OR) was used to describe effect size, with the control group as the reference category. An $\alpha$-level of 0.05 (twotailed) or $95 \%$ confidence interval (CI) was used to determine statistical significance.

The current dataset was large enough to show a significant difference for individuals (while not taking the nurse and class levels into account) of, for example, $8 \%$ for awareness of inadequate fruit intake and 0.17 for the mean servings of fruit intake per day, with a power of $80 \%$.

\section{Results}

\section{Participants}

Of the 495 children with consent, 486 children (98\%) participated in the baseline measurement including the Internet-tailored advice, of which 263 children belonged to the intervention group and 223 children to the control group. The post-test measurement included 469 children (97\% of baseline participation). The main reason for absence was illness.

Table 1 presents the characteristics of the children at baseline. There were no significant differences between the intervention and control groups regarding sociodemographic characteristics and baseline measures $(P \geq 0.05)$. Neither did the children who dropped out at post-test differ on sociodemographic characteristics, baseline measures and study group, compared with the children included at post-test $(P \geq 0.05)$.

\section{Outcome measures at post-test}

Fruit and vegetable intake did not differ significantly between the intervention and control groups (Tables 2 and 3). Of the secondary outcome measures, children in the intervention group were three times more likely to be aware of their personal inadequate fruit intake level at post-test than children in the control group (Table 2). Children in the intervention group were almost three times more likely to know the recommended vegetable levels at post-test than children in the control group (Table 3).

Table 1 Characteristics of the children at baseline by study group

\begin{tabular}{|c|c|c|c|}
\hline & $\begin{array}{c}\text { Intervention } \\
\text { group }(n=263)\end{array}$ & $\begin{array}{c}\text { Control } \\
\text { group }(n=223)\end{array}$ & $P$-value \\
\hline Age (years) & $10.3(0.5)^{*}$ & $10.3(0.5)^{*}$ & 0.65 \\
\hline Gender, boys (\%) & 45.6 & 48.9 & 0.32 \\
\hline Family status, two-parent family (\%) & 91.3 & 95.8 & 0.14 \\
\hline Ethnicity, Dutch background (\%)† & 88.6 & 84.6 & 0.19 \\
\hline Fruit frequency previous week (servings per day) & $1.1(0.8)^{\star}$ & $1.2(0.7)^{*}$ & 0.53 \\
\hline Awareness of inadequate fruit intake previous week (\%) & 25.9 & 18.0 & 0.09 \\
\hline 24-Hour recall vegetable intake $(\mathrm{g})$ & $76.9(66.7)^{*}$ & $77.9(64.6)^{\star}$ & 0.25 \\
\hline Awareness of inadequate vegetable intake previous day (\%) & 27.8 & 22.4 & 0.10 \\
\hline
\end{tabular}

${ }^{*}$ Mean (standard deviation).

† Both parents born in The Netherlands. 
Table 2 Self-reported fruit intake and determinants at post-test compared between the intervention and control groups

\begin{tabular}{|c|c|c|c|c|}
\hline & $\begin{array}{l}\text { Intervention } \\
\text { group }(n=263)\end{array}$ & $\begin{array}{c}\text { Control } \\
\text { group }(n=223)\end{array}$ & $\mathrm{OR}^{\star}(95 \% \mathrm{Cl})$ & $\beta \dagger(95 \% \mathrm{Cl})$ \\
\hline \multicolumn{5}{|l|}{ Fruit intake } \\
\hline Fruit frequency previous week (servings per day) & $1.1(0.7) \ddagger$ & $1.2(0.7) \ddagger$ & NA & $-0.05(-0.16,0.06)$ \\
\hline Usual daily fruit intake (\%) & 32.0 & 34.7 & $0.82(0.45,1.49)$ & NA \\
\hline \multicolumn{5}{|l|}{ Behavioural determinants } \\
\hline $\begin{array}{l}\text { Knowledge of recommendations, correct or higher } \\
\text { amount (\%) }\end{array}$ & 57.1 & 50.7 & $1.30(0.85,1.99)$ & NA \\
\hline Awareness of inadequate intake previous week (\%) & 30.8 & 13.0 & $3.04(1.75,5.26)$ & NA \\
\hline Self-efficacy: daily fruit intake achievable (\%) & 76.0 & 79.5 & $0.83(0.52,1.31)$ & NA \\
\hline Liking many types of fruit (\%) & 73.4 & 76.7 & $0.73(0.38,1.39)$ & NA \\
\hline Availability, always fruit at home (\%) & 74.5 & 77.6 & $0.89(0.53,1.51)$ & NA \\
\hline
\end{tabular}

OR - odds ratio; $\mathrm{Cl}$ - confidence interval; NA - not applicable.

* OR derived from multilevel logistic regression analyses adjusted for region, gender, age and baseline measure (except for knowledge and self-efficacy) and allowing a random intercept for nurse and class level.

†Unstandardised regression coefficient derived from multilevel linear regression analyses adjusted for region, gender, age and baseline measure (except for knowledge and self-efficacy) and allowing a random intercept for nurse and class level.

$\ddagger$ Mean (standard deviation).

Awareness of inadequate vegetable intake and knowledge of the fruit recommendation showed ORs in favour of the intervention group, but these were not significant. All remaining behavioural determinants of fruit and vegetable intake showed OR close to one.

\section{Exposure to intervention}

After receiving the Internet-tailored advice, 210 of the 263 children in the intervention group registered whether or not they had read the advice concerning fruit/vegetables. This resulted in $84 \%$ reporting that they had read both sets of advice completely, $8 \%$ had read part of the advice, $5 \%$ had read only the fruit or only the vegetable advice, and 3\% had read nothing. Based on the nurses' registrations, 240 (91\% of 263) children and one of their parents received at least some of the counselling. The nurses indicated that most of the counselling sessions concluded with encouraging the child's self-efficacy to change behaviour (about
42\%) and with stimulating goal setting (about 21\%). This indicates that for most of the children and parents in the intervention group at least knowledge of recommendations, awareness of personal intake, motivation to change and barriers to increase self-efficacy had been discussed.

\section{Discussion}

This cluster-randomised study indicated that an intervention combining Internet-tailored nutrition advice with dietary counselling by a nurse among elementary-school children resulted in better knowledge of the recommended vegetable intake levels and awareness of inadequate fruit intake, but no effects were found regarding actual intake levels. We note that, although effects were found for awareness, there were in fact some (non-significant) differences in awareness at baseline between the intervention and control groups.

Table 3 Self-reported vegetable intake and determinants at post-test compared between the intervention and control groups

\begin{tabular}{|c|c|c|c|c|}
\hline & $\begin{array}{c}\text { Intervention } \\
\text { group }(n=263)\end{array}$ & $\begin{array}{c}\text { Control } \\
\text { group }(n=223)\end{array}$ & $\mathrm{OR}^{\star}(95 \% \mathrm{Cl})$ & $\beta \dagger(95 \% \mathrm{Cl})$ \\
\hline \multicolumn{5}{|l|}{ Vegetable intake } \\
\hline 24-Hour recall vegetable intake $(\mathrm{g})$ & $76.9(68.7) \ddagger$ & $74.9(69.7) \ddagger$ & NA & $3.55(-7.90,15.00)$ \\
\hline Usual daily vegetable intake (\%) & 28.1 & 28.7 & $0.81(0.44,1.47)$ & NA \\
\hline \multicolumn{5}{|l|}{ Behavioural determinants } \\
\hline $\begin{array}{l}\text { Knowledge of recommendations, correct or higher } \\
\text { amount }(\%)\end{array}$ & 41.7 & 21.4 & $2.71(1.79,4.11)$ & NA \\
\hline Awareness of inadequate intake previous day (\%) & 31.9 & 24.2 & $1.42(0.87,2.34)$ & NA \\
\hline Self-efficacy, daily vegetable intake achievable (\%) & 60.5 & 59.5 & $0.98(0.58,1.66)$ & NA \\
\hline Liking many types of vegetables (\%) & 38.8 & 41.7 & $0.92(0.60,1.42)$ & NA \\
\hline $\begin{array}{l}\text { Availability, usually two or more types of } \\
\text { vegetables at dinner (\%) }\end{array}$ & 52.5 & 55.2 & $0.96(0.63,1.44)$ & NA \\
\hline
\end{tabular}

OR - odds ratio; $\mathrm{Cl}$ - confidence interval; NA - not applicable.

*OR derived from multilevel logistic regression analyses adjusted for region, gender, age and baseline measure (except for knowledge and self-efficacy) and allowing a random intercept for nurse and class level.

† Unstandardised regression coefficient derived from multilevel linear regression analyses adjusted for region, gender, age and baseline measure (except for knowledge and self-efficacy) and allowing a random intercept for nurse and class level.

$\ddagger$ Mean (standard deviation). 
We are not aware of any other controlled studies on similar interventions. A non-controlled study on a similar intervention among adolescents in primary care settings in the USA reported indications of increased fruit and vegetable intakes ${ }^{26}$.

Our two-component intervention was compact and embedded in the existing practice of the preventive health care at schools, offering the benefit of a large reach and increasing the chance of future implementation. Counselling the child in the presence of one parent enabled involvement of the family; this is important because the social environment is a recognised facilitator for dietary behaviour ${ }^{9,10,27}$. The study had a high response rate (after receiving consent to participate from the parents) and had a high level of exposure to the intervention. Potentially, once taken out of the research mode and implemented in standard practice, there may be an even higher participation rate since a less strict informed consent procedure would be applied than in our study. Our sample was a representative study population, as it does not differ substantially in sociodemographic characteristics (gender, family status and ethnicity) from the general Dutch adolescent population when compared with nationwide data on schoolchildren in elementary schools ${ }^{28}$.

A possible limitation concerning the measures was the application of child self-reports. Self-administered questionnaires can be subject to response bias (socially desirable answers), thereby resulting in overreporting of healthy behaviour or positive beliefs. Unfortunately, the use of more reliable and valid measures often incurs greater costs and longer time investment, as well as increasing the burden for the participants (see also further below).

That only one outcome measurement (i.e. 3 months after the baseline measurement) was applied may be considered a limitation. Because our two-component intervention was very compact, perhaps only short-term effects of intake levels were present, which we did not measure. In future studies outcome measurements should be made at several points in time, including short-term outcome measurements.

We now discuss some possible explanations for the absence of effects of our two-component intervention regarding dietary intake, and make suggestions for improvement. Although Patrick et al. ${ }^{26}$ added mail and telephone contact to the two-component approach, this did not result in improved intakes. In contrast to that study, multi-component interventions with longer duration and more contact hours generally achieve better results than shorter programmes ${ }^{9,10}$. The current Internet-based twocomponent intervention may be regarded as an initial part of a broader approach, involving repetition of the current components or the addition of other components ${ }^{8,10}$. Furthermore, to improve effectiveness regarding intake levels, the current intervention components may need improvement. For instance, relying on the vegetable intake of the previous day is generally regarded as valid, but may have some limitations. For example, assessments that took place on Mondays might have resulted in lower reports of vegetable intake; in The Netherlands, Sundays are 'easy or no cooking days', as confirmed by Wind et $a l .{ }^{17}$. When parents or children 'feel' that their assessment and therefore their personal advice is not representative, the advice may lack a positive effect. More comprehensive assessment may improve this, but this will also increase the burden on the respondents. Another solution may be to focus more on the advice and counselling concerning the relevance of eating sufficient vegetables 'every day'. Additionally, the nurses reported that some children of this age may have a poor recognition of vegetables, e.g. not knowing whether the food was a vegetable or some other type of food, or to which category the type of vegetable in question belonged to (e.g. heated, salad or mixed). This has also been indicated in previous studies $^{10,29}$. Adding pictures and more explanation about the questions in the assessment as well as improvement regarding the wording of questions might avoid this. An alternative approach might be more advice on how to recognise vegetables ${ }^{19}$. More research is needed to test whether these suggestions would indeed improve the effect on vegetable intake.

In conclusion, this study was unique for its school-based intervention integrated within preventive health care. The dual approach of advice and counselling guaranteed a repetition of the main health message, combined written and oral feedback, and involved both the school and home environment. Linking the two components by means of the Internet allowed automatic support of the dietary counselling, thus enhancing efficiency. The study showed that this compact, integrated two-component intervention induced positive changes in knowledge and awareness of dietary intake levels among schoolchildren. To induce changes in intake levels, more comprehensive interventions may be needed.

\section{Acknowledgements}

The authors thank the employees of the municipal health services in Vlaardingen and Harderwijk, the schools, the schoolchildren and their parents/guardians for their enthusiastic participation; Halime Kaya-Dag for data collection and other supportive tasks; and Saskia te Velde for helping with the analyses. The Netherlands Organisation for Health Research and Development (ZonMw) \#4010.0011 funded this research.

\section{References}

1 Maynard M, Gunnell D, Emmett P, Frankel S, Davey Smith G. Fruit, vegetables, and antioxidants in childhood and risk of adult cancer: the Boyd Orr cohort. Journal of Epidemiology and Community Health 2003; 57(3): $218-25$. 
2 Paolini M, Sapone A, Canistro D, Antonelli MA, Chieco P. Diet and risk of cancer. Lancet 2003; 361(9353): 257-8.

3 World Health Organization (WHO). Diet, Nutrition and the Prevention of Chronic Diseases. Technical Report Series No. 916. Geneva: WHO, 2003; i-viii, 1-149.

4 Hung HC, Joshipura KJ, Jiang R, Hu FB, Hunter D, SmithWarner SA, et al. Fruit and vegetable intake and risk of major chronic disease. Journal of National Cancer Institute 2004; 96(21): 1577-84

5 Yngve A, Wolf A, Poortvliet E, Elmadfa I, Brug J, Ehrenblad B, et al. Fruit and vegetable intake in a sample of 11-year-old children in 9 European countries: The Pro Children Crosssectional Survey. Annals of Nutrition \& Metabolism 2005; 49(4): 236-45.

6 Lien N, Lytle LA, Klepp KI. Stability in consumption of fruit, vegetables, and sugary foods in a cohort from age 14 to age 21. Preventive Medicine 2001; 33(3): 217-26.

7 Mikkila V, Rasanen L, Raitakari OT, Pietinen P, Viikari J. Longitudinal changes in diet from childhood into adulthood with respect to risk of cardiovascular diseases: The Cardiovascular Risk in Young Finns Study. European Journal of Clinical Nutrition 2004; 58(7): 1038-45.

8 Doak C. Large-scale interventions and programmes addressing nutrition-related chronic diseases and obesity: examples from 14 countries. Public Health Nutrition 2002; 5(1A): 275-7.

9 Blanchette L, Brug J. Determinants of fruit and vegetable consumption among 6-12-year-old children and effective interventions to increase consumption. Journal of Human Nutrition and Dietetics 2005; 18(6): 431-43.

10 Shepherd J, Harden A, Rees R, Brunton G, Garcia J, Oliver S, et al. Young people and healthy eating: a systematic review of research on barriers and facilitators. Health Education Research 2006; 21(2): 239-57.

11 Brug J, Campbell M, van Assema P. The application and impact of computer-generated personalized nutrition education: a review of the literature. Patient Education and Counseling 1999; 36(2): 145-56.

12 Brug J, Oenema A, Campbell M. Past, present, and future of computer-tailored nutrition education. American Journal of Clinical Nutrition 2003; 77(Suppl. 4): $1028 \mathrm{~S}-34 \mathrm{~S}$.

13 Mangunkusumo RT, Brug J, Duisterhout JS, de Koning HJ, Raat H. Feasibility, acceptability, and quality of Internetadministered adolescent health promotion in a preventivecare setting. Health Education Research 2006 May 19; Epub ahead of print.

14 Kroeze W, Werkman A, Brug J. A systematic review of randomized trials on the effectiveness of computertailored education on physical activity and dietary behaviors. Annals of Behavioral Medicine 2006; 31(3): 205-23.

15 Bere E, Klepp KI. Correlates of fruit and vegetable intake among Norwegian schoolchildren: parental and self-reports. Public Health Nutrition 2004; 7(8): 991-8.
16 Bere E, Klepp KI. Changes in accessibility and preferences predict children's future fruit and vegetable intake. International Journal of Behavioral Nutrition and Physical Activity 2005; 2: 15.

17 Wind M, Bobelijn K, De Bourdeaudhuij I, Klepp KI, Brug J. A qualitative exploration of determinants of fruit and vegetable intake among 10- and 11-year-old schoolchildren in the low countries. Annals of Nutrition \& Metabolism 2005; 49(4): 228-35

18 Wind M, De Bourdeaudhuij I, Te Velde S, Sandvik C, Due P, Klepp KI, et al. Correlates of fruit and vegetable consumption among 11-year-old Belgian-Flemish and Dutch schoolchildren. Journal of Nutrition Education and Behaviour 2006; 38(4): 211-21.

19 Perez-Rodrigo C, Wind M, Hildonen C, Bjelland M, Aranceta J, Klepp KI, et al. The pro children intervention: applying the intervention mapping protocol to develop a school-based fruit and vegetable promotion programme. Annals of Nutrition \& Metabolism 2005; 49(4): 267-77.

20 Wiggers LC, Oort FJ, Dijkstra A, de Haes JC, Legemate DA, Smets EM. Cognitive changes in cardiovascular patients following a tailored behavioral smoking cessation intervention. Preventive Medicine 2005; 40(6): 812-21.

21 Campbell MK, Elbourne DR, Altman DG. CONSORT statement: extension to cluster randomised trials. British Medical Journal 2004; 328(7441): 702-8.

22 Keij I. Aantallen allochtonen volgens verschillende definities [Number of Foreigners According to Different Definitions]. Voorburg/Heerlen: Centraal Bureau voor de Statistiek (Statistics Netherlands), 2000.

23 Van Assema P, Brug J, Ronda G, Steenhuis I, Oenema A A short Dutch questionnaire to measure fruit and vegetable intake: relative validity among adults and adolescents. Nutrition and Health 2002; 16(2): 85-106.

24 Twisk JWR, ed. Missing data in longitudinal studies In: Applied Longitudinal Data Analysis for Epidemiology. Cambridge: Cambridge University Press, 2003; 202-24.

25 Rasbash J, Steele F, Browne W, Prosser B. A User's Guide to MLwiN Version 2.0. Bristol: University of Bristol, 2005.

26 Patrick K, Sallis JF, Prochaska JJ, Lydston DD, Calfas KJ, Zabinski MF, et al. A multicomponent program for nutrition and physical activity change in primary care: PACE + for adolescents. Archives of Pediatrics \& Adolescent Medicine 2001; 155(8): 940-6.

27 Vereecken CA, Keukelier E, Maes L. Influence of mother's educational level on food parenting practices and food habits of young children. Appetite 2004; 43(1): 93-103.

28 Statistics Netherlands. Statline [online]. Available at http://statline.cbs.nl/StatWeb/start.asp?lp=Search/Search. Accessed 18 October 2005.

29 Edwards JS, Hartwell HH. Fruit and vegetables - attitudes and knowledge of primary school children. Journal of Human Nutrition and Dietetics 2002; 15(5): 365-74. 